\title{
SHEAR INDUCED REDISTRIBUTION OF FLUID WITHIN A UNIFORMLY SWOLLEN NONLINEAR ELASTIC CYLINDER
}

\author{
A. WINEMAN \\ Department of Mechanical Engineering and Applied Mechanics, The University of Michigan, \\ Ann Arbor, MI 48109 , U.S.A. \\ K. R. RAJAGOPAL \\ Department of Mechanical Engineering, The University of Pittsburgh, Pittsburgh, PA 15101, U.S.A.
}

\begin{abstract}
The theory of mixtures is applied to the determination of equilibrium states of a solid-fluid mixture which is isolated from contact with a fluid bath. In the particular problem considered, a hollow rubber cylinder first undergoes unconstrained swelling in a fluid bath. The solid is homogeneously deformed to a larger cylindrical shape, the fluid is uniformly dispersed and the mixture is in a saturated equilibrium state. The mixture is then bonded to rigid impermeable membranes at its inner and outer surfaces. Rigid impermeable flat plates restrain motion at its ends. While the swollen length is held fixed, relative rotation of the membranes induces shear distortion in the rubber-fluid mixture. The resulting normal stresses cause a change in the mixture from its initial equilibrium state in which the system is homogeneously swollen but unsheared to a new equilibrium state in which there is radial variation of both the solid deformation and fluid density. A numerical example, using properties for a particular rubber-fluid mixture, shows that the volume of the mixture and the fluid density decrease near the inner wall of the cylinder and increase near the outer wall.
\end{abstract}

\section{INTRODUCTION}

When a rubbery material is placed in contact with an appropriate liquid, the liquid will diffuse into the rubber. There is a very marked interaction between the liquid and the rubber. The solid will undergo a large deformation consisting of both swelling and distortion induced by stresses. The deformation of the solid will affect the distribution of the liquid. This interaction is characteristic of other important situations, such as the diffusion of moisture into a composite material or of a biological fluid into biotissue.

The proper framework for studying such phenomena is the theory of interacting continua, also known as the theory of mixtures. In a series of papers (see for example [1-3]), Rajagopal, Wineman and co-workers have applied this theory to the study of a number of aspects of the interaction between a fluid and a highly elastic solid. A summary of this work is presented in a recent review article [4]. Mass flux-pressure differences were studied in [1-3]. Problems involving an equilibrium state in which a non-homogeneously deformed cylinder is in continuous contact with a fluid bath were treated in [5]. The present work is concerned with equilibrium states of a solid-fluid mixture which is isolated from contact with a fluid bath.

Consider a hollow cylinder of rubber which is immersed in a liquid bath and allowed to undergo unconstrained swelling. The cylinder-liquid mixture will reach a swollen saturated equilibrium state in which the cylinder has become homogeneously deformed and contains uniformly dispersed liquid. The mixture is removed from the bath and is bonded to rigid impermeable cylinders at its inner and outer surfaces. Impermeable rigid plates are attached at its end in order to maintain a plane deformation. The outer cylinder is then rotated relative to the inner cylinder about the center line of the system, thereby subjecting the rubber-liquid mixture to radially dependent shear deformation. The normal stresses which accompany the shear can be expected to have the following consequences. First, the rubber becomes non-homogeneously stretched and swollen as well as sheared, and secondly the fluid becomes non-homogeneously distributed throughout the system. The purpose is to determine these new 
distributions once the system has reached equilibrium. The time dependent redistribution process is also of great interest and will be the subject of future work.

The general theory of interacting continua has been presented in review papers by Bowen [6] and Atkin and Craine [7]. The fundamental quantities and field equations which are relevant to this work are outlined in Section 2. Constitutive equations for a solid-liquid mixture in an equilibrium state are given in Section 3. An equation which characterizes saturation is introduced in Section 4. Section 5 contains a reduced system of equations which is obtained by eliminating certain variables. The swollen unconstrained state of the cylindrical mixture and an equation for its new dimensions is introduced in Section 6. Section 7 contains the formulation of the boundary value problem for the sheared state of a general swollen cylinder. A particular set of material properties and the corresponding form of the boundary value problem is presented in Section 8. Finally, results of the numerical solution of the governing equations are discussed in Section 9.

\section{FUNDAMENTAL QUANTITIES AND EQUATIONS}

The concepts and equations of the theory of mixtures which apply to the interaction of an ideal fluid and an elastic solid have been presented in [1-4]. The notation and equations which are essential to the present study are summarized here.

The positions of a solid particle in its reference and current configurations will be denoted, respectively, by $\mathbf{X}^{\mathrm{s}}$ and $\mathbf{x}^{\mathrm{s}}$. Its deformation gradient is

$$
\mathbf{F}=\frac{\partial \mathbf{x}^{\mathrm{s}}}{\partial \mathbf{X}^{\mathrm{s}}}
$$

Let $\rho_{0}^{\mathrm{s}}$ denote the density of the solid in its unmixed state (mass per unit unmixed volume), and $\rho^{s}$ denote its density in the mixture (mass per unit volume of the mixture). The corresponding quantities for the fluid are $\rho_{0}^{\mathrm{f}}$ and $\rho^{\mathrm{f}}$, respectively. At each point in the mixture region, the mass density of the mixture is

$$
\rho=\rho^{\mathrm{s}}+\rho^{\mathrm{f}} .
$$

The partial stress tensors associated with the solid and fluid will be denoted, respectively, by $\sigma^{s}$ and $\sigma^{\mathrm{f}}$. The total stress in the mixture is

$$
\sigma=\sigma^{\mathrm{s}}+\sigma^{\mathrm{f}}
$$

The equations which represent physical principles and which are relevant to the present work are:

\subsection{Conservation of mass $\rightarrow$ solid}

$$
\rho^{\mathrm{s}} \operatorname{det} \mathbf{F}=\rho_{0}^{\mathrm{s}} .
$$

The conservation of mass equation for the fluid will play no role here and is therefore omitted.

It is assumed that there is no external body force. The interactive body force which each constituent exerts on the other is denoted by $b$.

\subsection{Equilibrium equation-solid}

$$
\operatorname{div} \sigma^{s}-\mathbf{b}=0
$$

\subsection{Equilibrium equation-fluid}

$$
\operatorname{div} \sigma^{\mathbf{r}}+\mathbf{b}=\mathbf{0}
$$

It is a consequence of the law of balance of moment of momentum that the total stress is symmetric, $\sigma=\sigma^{\mathrm{T}}$, but that the partial stress need not be. 


\section{CONSTITUTIVE EQUATIONS}

It is assumed that when a volume of fluid is mixed with a volume of solid, the volume of the mixture is the sum of the unmixed volumes. This assumption of volume additivity is commonly made when an organic liquid mixes with rubber [8]. This volume additivity assumption imposes the following constraint on the densities of the solid and fluid (cf. Mills [9]).

$$
\frac{\rho^{\mathrm{s}}}{\rho_{0}^{\mathrm{s}}}+\frac{\rho^{\mathrm{f}}}{\rho_{0}^{\mathrm{f}}}=1
$$

Let $A_{0}$ denote the Helmholtz free energy of the mixture per unit volume of the solid in its reference state. Let it be assumed that the solid is isotropic in its reference state. It can be shown that $A_{0}=A_{0}\left(I_{1}, I_{2}, \rho^{\mathrm{f}}\right)$. Here $I_{1}$ and $I_{2}$ are the invariants of the left Cauchy-Green deformation tensor $\mathbf{B}=\mathbf{F F}^{\mathrm{T}}$,

$$
I_{1}=\operatorname{tr} \mathbf{B}, \quad I_{2}=\frac{1}{2}\left[I_{1}^{2}-\operatorname{tr}\left(\mathbf{B}^{2}\right)\right] .
$$

The constitutive equations for the partial stresses in the equilibrium state are given by

$$
\begin{gathered}
\sigma^{\mathrm{s}}=-p \frac{\rho^{\mathrm{s}}}{\rho_{0}^{\mathrm{s}}} \mathbf{I}+2 \frac{\rho^{\mathrm{s}}}{\rho_{0}^{\mathrm{s}}}\left[\left(\frac{\partial A_{0}}{\partial I_{1}}+I_{1} \frac{\partial A_{0}}{\partial I_{2}}\right) \mathbf{B}-\frac{\partial A_{0}}{\partial I_{2}} \mathbf{B}^{2}\right], \\
\sigma^{\mathrm{f}}=-p \frac{\rho^{\mathrm{f}}}{\rho_{0}^{\mathrm{f}}} \mathbf{I}-\rho \rho^{\mathrm{f}} \frac{\partial}{\partial \rho^{\mathrm{f}}}\left(\frac{1}{\rho} \frac{\rho^{\mathrm{s}}}{\rho_{0}^{\mathrm{s}}} A_{0}\right) \mathbf{I},
\end{gathered}
$$

and for the interactive body force by

$$
\begin{aligned}
b_{i}= & -p \frac{\partial}{\partial x_{i}}\left(\frac{\rho^{\mathrm{s}}}{\rho_{0}^{\mathrm{s}}}\right)+\rho^{\mathrm{s}} \frac{\partial}{\partial \rho^{\mathrm{f}}}\left(\frac{\rho^{\mathrm{s}}}{\rho_{0}^{\mathrm{s}}} \frac{1}{\rho} A_{0}\right) \frac{\partial \rho^{\mathrm{f}}}{\partial x_{i}} \\
& -\frac{\rho^{\mathrm{f}}}{\rho} \frac{\rho^{\mathrm{s}}}{\rho_{0}^{\mathrm{s}}}\left[\left(\frac{\partial A_{0}}{\partial I_{1}}+I_{1} \frac{\partial A_{0}}{\partial I_{2}}\right) \delta_{k l}-\frac{\partial A_{0}}{\partial I_{2}} B_{k l}\right] \frac{\partial B_{k l}}{\partial x_{i}} .
\end{aligned}
$$

In (9)-(11), $p$ is an indeterminate scalar which arises from the volume additivity constraint (7).

\section{SATURATION EQUATION OF STATE}

Let a block of the unstrained unswollen solid be placed in a bath of the fluid and let a uniform surface traction distribution be applied to its surface. The block will swell with the fluid and become distorted by the surface tractions. The solid-fluid mixture will eventually reach an equilibrium state in which the solid is homogeneously deformed and contains uniformly dispersed liquid. No additional fluid will enter the block and a saturated state is reached. This state can be characterized by a condition presented in [8]. In [11], Rajagopal $e t$ al. expressed this condition in a form which is appropriate for the theory of mixtures. Let the total stress tensor in the saturated state be denoted by $\sigma^{\text {sat }}$. The saturation equation of state which relates the stress, solid deformation and fluid density is given by

$$
\sigma^{\mathrm{sat}}=\rho_{0}^{\mathrm{f}}\left(\frac{\rho^{\mathrm{s}}}{\rho_{0}^{\mathrm{s}}}\right)^{2} \frac{\partial A_{0}}{\partial \rho^{\mathrm{f}}} \mathbf{I}+2 \frac{\rho^{2}}{\rho_{0}^{\mathrm{s}}}\left[\left(\frac{\partial A_{0}}{\partial I_{1}}+I_{1} \frac{\partial A_{0}}{\partial I_{2}}\right) \mathbf{B}-\frac{\partial A_{0}}{\partial I_{2}} \mathbf{B}^{2}\right]
$$

\section{REDUCED SYSTEM OF EQUATIONS}

A reduced system of equations can be obtained by eliminating the unknown scalar $p$ from the governing system of equations. If equilibrium equations (5) and (6) are added, the result is 
an equation in terms of the total stress (3),

$$
\operatorname{div} \sigma=0 .
$$

Next substitute (9) and (11) into (5) and (7), (9), (10) into (13). It is possible to eliminate the scalar $p$ between these equations and, after straightforward but lengthy manipulations, reduce the system of equations to the form

$$
\operatorname{div} \sigma^{\text {sat }}=\mathbf{0} \text {, }
$$

in which $\sigma^{\text {sat }}$ is given by (12) (cf. Gandhi and Usman [12]).

\section{UNCONSTRAINED SWELLING OF A CYLINDER}

Consider an isotropic nonlinear elastic solid whose reference shape is a hollow circular cylinder. Its inner and outer radii are $R_{\mathrm{i}}$ and $R_{\mathrm{o}}$, respectively, and its length is $L_{0}$. Let a cylindrical coordinate system be concentric with the elastic cylinder and the coordinates of a particle of the solid in this system by $(R, \Theta, Z)$.

Let the cylinder be immersed in a fluid bath so that it can swell freely, that is, without the application of surface tractions. It can be anticipated that the cylinder will reach a saturated state in which it is uniformly swollen. If the coordinates of a particle in this state are denoted by $(\hat{r}, \hat{\theta}, \hat{z})$, the swollen configuration can be described by the mapping

$$
\begin{aligned}
& \hat{r}=\lambda R, \\
& \hat{\theta}=\Theta, \\
& \hat{z}=\lambda Z,
\end{aligned}
$$

where $\lambda$, the stretch ratio for uniform swelling, is a constant. From (1), (4), (7), (8) and (15), it is found that

$$
\begin{array}{r}
\mathbf{F}=\lambda \mathbf{I}, \quad \mathbf{B}=\mathbf{F F}^{\mathrm{T}}=\lambda^{2} \mathbf{I}, \quad \mathbf{B}^{2}=\lambda^{4} \mathbf{I}, \\
\operatorname{det} \mathbf{F}=\lambda^{3}, \quad I_{1}=3 \lambda^{2}, \quad I_{2}=3 \lambda^{4}, \\
\frac{\rho^{\mathrm{s}}}{\rho_{0}^{\mathrm{s}}}=\frac{1}{\lambda^{3}}, \quad \frac{\rho^{\mathrm{f}}}{\rho_{0}^{\mathrm{f}}}=1-\frac{1}{\lambda^{3}} .
\end{array}
$$

Let these be substituted into the saturation equation of state (12) in which $\sigma^{\text {sat }}=\mathbf{0}$. This gives the following equation for $\lambda$,

$$
\frac{\rho_{0}^{\mathrm{f}}}{\lambda^{3}} \frac{\partial A_{0}}{\partial \rho^{\mathrm{f}}}+2 \lambda^{2}\left[\frac{\partial A_{0}}{\partial I_{1}}+2 \lambda^{2} \frac{\partial A_{0}}{\partial I_{2}}\right]=0 .
$$

\section{CIRCUMFERENTIAL SHEARING OF THE SWOLLEN CYLINDER}

Let the swollen cylinder be bonded to a fixed impermeable rigid cylindrical support at its inner surface of radius $\lambda R_{\mathrm{i}}$, and let an impermeable rigid mombrane be applied to its outer surface of radius $\lambda R_{\mathrm{o}}$. Also, let impermeable rigid plates be applied to the ends of the cylinder. A moment acting about the cylinder center line is applied to the membrane, whose purpose is to transmit a uniformly distributed circumferential shear stress distribution to the outer surface of the swollen cylinder. Let $M$ denote the moment per unit length of the cylinder. The deformation of the cylinder will be described by the mapping,

$$
\begin{aligned}
& r=f(\hat{r})=f(\lambda R), \\
& \theta=\hat{\theta}+g(\hat{r})=\Theta+g(\lambda R), \\
& z=\hat{z}+\lambda Z,
\end{aligned}
$$


where $\hat{r} \ni\left[\lambda R_{\mathrm{i}}, \lambda R_{\mathrm{o}}\right]$. Here, $g(\hat{r})$ is the rotation of the cylindrical surface at radius $\hat{r}$ and accounts for local shear deformation. $f(\hat{r})$ gives the radial motion of the cylindrical surface due to normal stresses which arise during shear. The radial deformation can be non-uniform and will be accompanied by the redistribution of fluid within the cylinder. The third equation of (18) expresses the condition that the cylinder is maintained at a fixed length as the moment is applied.

The deformation gradient (1) can be written as

$$
\mathbf{F}=\lambda \hat{\mathbf{F}},
$$

where $\hat{\mathbf{F}}$ denotes the deformation gradient with respect to the swollen unsheared configuration. It is given with respect to cylindrical coordinates by

$$
\hat{\mathbf{F}}=\left[\begin{array}{ccc}
\mathrm{d} r / \mathrm{d} \hat{r} & 0 & 0 \\
r \mathrm{~d} \theta / \mathrm{d} \hat{r} & r / \hat{r} & 0 \\
0 & 0 & 1
\end{array}\right]=\left[\begin{array}{ccc}
f^{\prime} & 0 & 0 \\
f g^{\prime} & f / \hat{r} & 0 \\
0 & 0 & 1
\end{array}\right],
$$

where the prime denotes differentiation with respect to the argument $\hat{r}$. Let

$$
\hat{\lambda}=r / \hat{r}=f(\hat{r}) / \hat{r}
$$

which represents the ratio of the lengths of a circular line in its current and swollen unsheared configurations. The ratio of the volume of a material element of the solid in the current configuration to its volume in the unswollen unsheared state is

$$
\operatorname{det} \mathbf{F}=\lambda^{3} \hat{J}, \quad \hat{J}=\hat{\lambda} f^{\prime},
$$

in which $\lambda^{3}$ is the ratio of the volume in the swollen unsheared state to its initial volume, and is the same for all material elements. $\hat{J}$ is the ratio of the current volume to its volume in the swollen unsheared configuration, and will vary with radius.

The invariants $(8)$ become

$$
\begin{aligned}
& I_{1}=\lambda^{2}\left[1+\left(f^{\prime}\right)^{2}+\lambda^{2}+\left(f g^{\prime}\right)^{2}\right], \\
& I_{2}=\lambda^{4}\left[\left(1+\left(f^{\prime}\right)^{2}\right) \hat{\lambda}^{2}+\left(f^{\prime}\right)^{2}+\left(f g^{\prime}\right)^{2}\right] .
\end{aligned}
$$

Using (4), (7) and (22), the density ratios for the solid and fluid are, respectively,

$$
\begin{aligned}
& \rho^{\mathrm{s}} / \rho_{0}^{\mathrm{s}}=\frac{1}{\lambda^{3} \hat{\jmath}}, \\
& \rho^{\mathrm{f}} / \rho_{0}^{\mathrm{f}}=1-\frac{1}{\lambda^{3} \hat{\jmath}} .
\end{aligned}
$$

Dependence on $\hat{J}$ accounts for the redistribution of fluid due to shear. If $\hat{J}$ increases, then (24) shows that $\rho^{\mathrm{f}} / \rho_{0}^{\mathrm{f}}$ also increases.

The normal stress components calculated from (12) are

$$
\begin{gathered}
\sigma_{r r}^{\mathrm{sat}}=\Phi+2\left(\frac{\rho^{\mathrm{s}}}{\rho_{0}^{\mathrm{s}}}\right) \lambda^{2}\left(f^{\prime}\right)^{2}\left[\frac{\partial A_{0}}{\partial I_{1}}+\lambda^{2}\left(1+\hat{\lambda}^{2}\right) \frac{\partial A_{0}}{\partial I_{2}}\right], \\
\sigma_{\theta \theta}^{\mathrm{sat}}=\Phi+2\left(\frac{\rho^{\mathrm{s}}}{\rho_{0}^{\mathrm{s}}}\right) \lambda^{2}\left[\left(\frac{\partial A_{0}}{\partial I_{1}}+\lambda^{2} \frac{\partial A_{0}}{I_{2}}\right)\left(\hat{\lambda}^{2}+\left(f g^{\prime}\right)^{2}\right)+\lambda^{2} \frac{\partial A_{0}}{\partial I_{2}}(f \hat{\lambda})^{2}\right], \\
\sigma_{z z}^{\mathrm{sat}}=\Phi+2 \frac{\rho^{\mathrm{s}}}{\rho_{0}^{\mathrm{s}}} \lambda^{2}\left[\frac{\partial A_{0}}{\partial I_{1}}+\lambda^{2}\left(\left(f^{\prime}\right)^{2}+\hat{\lambda}^{2}+\left(f g^{\prime 2}\right)\right) \frac{\partial A_{0}}{I_{2}}\right],
\end{gathered}
$$

where

$$
\Phi=\rho_{0}^{\mathrm{f}}\left(\frac{\rho^{\mathrm{s}}}{\rho_{0}^{\mathrm{s}}}\right)^{2} \frac{\partial A_{0}}{\partial \rho^{\mathrm{f}}}
$$


In addition,

$$
\sigma_{r \theta}^{\mathrm{sat}}=2 \frac{\rho^{\mathrm{s}}}{\rho_{0}^{\mathrm{s}}} \lambda^{2}\left(f g^{\prime} f^{\prime}\right)\left[\frac{\partial A_{0}}{\partial I_{1}}+\lambda^{2} \frac{\partial A_{0}}{I_{2}}\right],
$$

and $\sigma_{r z}^{\text {sat }}=\sigma_{\theta z}^{\text {sat }}=0$.

The equation of equilibrium in the radial direction is

$$
\frac{\mathrm{d} \sigma_{r r}^{\mathrm{sat}}}{\mathrm{d} r}+\frac{\sigma_{r r}^{\mathrm{sat}}-\sigma_{\theta \theta}^{\mathrm{sat}}}{r}=0,
$$

while that in the circumferential direction is

$$
\frac{\mathrm{d} \sigma_{r \theta}^{\mathrm{sat}}}{\mathrm{d} r}+\frac{2 \sigma_{r \theta}^{\mathrm{sat}}}{r}=0
$$

Note that the stress components are expressed in terms of functions of radius $\hat{r}$ in the swollen unsheared configuration. It will thus be convenient to transform the equilibrium equations to a form in which $\hat{r}$ is the independent variable. Equation (30) becomes

$$
\frac{\mathrm{d} \sigma_{r r}^{\mathrm{sat}}}{\mathrm{d} \hat{r}}+\frac{\hat{J}}{\hat{\lambda}^{2}} \frac{\sigma_{r r}^{\mathrm{sat}}-\sigma_{\theta \theta}^{\mathrm{sat}}}{\hat{r}}=0,
$$

and equation (31) becomes

$$
\frac{\mathrm{d} \sigma_{r \theta}^{\mathrm{sat}}}{\mathrm{d} \hat{r}}+2 \frac{\hat{J}}{\hat{\lambda}^{2}} \frac{\sigma_{r \theta}^{\mathrm{sat}}}{\hat{r}}=0
$$

in which use has been made of (22).

When the stresses given by (25), (26), (28) and (29) are substituted into (32) and (33) and use is made of (22), (23) and (28), the result is a system of second order nonlinear ordinary differential equations for $f(\hat{r})$ and $g(\hat{r})$. The solution depends on $\lambda$ and $M$ and must meet the boundary conditions

$$
\begin{gathered}
f\left(\lambda R_{\mathrm{i}}\right)=\lambda R_{\mathrm{i}}, \quad f\left(\lambda R_{\mathrm{o}}\right)=\lambda R_{\mathrm{o}}, \\
g\left(\lambda R_{\mathrm{i}}\right)=0 .
\end{gathered}
$$

\section{SPECIFIC $A_{0}$}

Consider materials for which $A_{0}$ has the form

$$
A_{0}=A_{0}^{\mathrm{m}}\left(\rho^{\mathrm{f}}\right)+A_{0}^{\mathrm{e}}\left(I_{1}, I_{2}\right) \text {. }
$$

$A_{0}^{\mathrm{m}}$ is the contribution to $A_{0}$ from the mixing of the fluid and solid and $A_{0}^{\mathrm{e}}$ is the contribution from the deformation of the solid. This is the Flory-Rehner assumption and is discussed in [8]. In particular, $A_{0}^{\mathrm{m}}$ is such that $\Phi$ in (28) has the form

$$
\Phi=\frac{R T}{V_{1}}\left[\ln (1-v)+v+\chi v^{2}\right]
$$

where

$$
v=\frac{1}{\lambda^{3} \hat{J}}
$$

and represents the volume fraction of the solid in the mixture. $A_{0}^{\mathrm{e}}$ is given by

$$
A_{0}^{\mathrm{e}}=\frac{1}{2} K\left[\left(I_{1}-3\right)+\alpha\left(I_{2}-3\right)\right], \quad K=\rho_{0}^{\mathrm{s}} \frac{R T}{M_{\mathrm{c}}},
$$


$R$ is the universal gas constant, $T$ is the absolute temperature, $V_{1}$ is the molar volume of the fluid, $M_{\mathrm{c}}$ is the molecular weight between cross links for the solid, and $\chi$ is an interaction constant which depends on the specific solid-fluid mixture, and $\alpha$ is a constant.

The development of the form for $A_{0}$ given by (36)-(39), with $\alpha=0$, is discussed in [8]. It is based on a combination of statistical and empirical arguments, and has some experimental justification. When this $A_{0}$ is substituted into (12), the result is a set of specific stress-stretchswelling relations for homogeneous deformations of saturated rubber-fluid mixtures. It is shown in [8] that predictions based on these relations are in good agreement with experimental results involving homogeneous triaxial deformations.

When $\alpha=0, A_{0}^{\mathrm{e}}$ in (39) corresponds to the neo-Hookean material in nonlinear elasticity. When $\alpha>0, A_{0}^{\text {e }}$ corresponds to a Mooney-Rivlin material. Parameter $\alpha$ is introduced in order to permit a study of the dependence of results on $I_{2}$.

Let $A_{0}$ in (36)-(39) be substituted into (17). Since this is the equation for the stretch ratio $\lambda$ for the swollen unsheared state, $\hat{J}=1$ in (38) and $v=1 / \lambda^{3}$. The resulting equation for $\lambda$ is

$$
\frac{M_{\mathrm{c}}}{\rho_{0}^{\mathrm{s}} V_{1}}\left[\ln \left(1-\frac{1}{\lambda^{3}}\right)+\frac{1}{\lambda^{3}}+\frac{\chi}{\lambda^{6}}\right]+\frac{1}{\lambda}\left(1+2 \lambda^{2} \alpha\right)=0
$$

Now consider the equations for the boundary value problem for the sheared state. Substitute $A_{0}$ into the expression for the shear stress (29), and make use of (22) and (24). This gives

$$
\sigma_{r \theta}^{\mathrm{sat}}=K\left(1+\lambda^{2} \alpha\right) \frac{f g^{\prime}}{\lambda \hat{\lambda}} .
$$

Next note that equilibrium equation (31) can be integrated to give

$$
\sigma_{r \theta}^{\text {sat }}=\frac{M}{2 \pi r^{2}}=\frac{M}{2 \pi \hat{r}^{2} \hat{\lambda}^{2}},
$$

where the last step follows from (21). Combining (41) and (42) leads to the following expression for $f g^{\prime}$,

$$
f g^{\prime}=\frac{M \lambda}{2 \pi K\left(1+\lambda^{2} \alpha\right)} \frac{1}{\hat{r}^{2} \hat{\lambda}}
$$

Consider the normal stresses $\sigma_{r r}^{\text {sat }}$ and $\sigma_{\theta \theta}^{\text {sat }}$. According to (37) and (38), $\Phi$ depends on $\hat{J}$. Since $\hat{J}$ represents the local volume change and is related to the fluid density redistribution by $(24), \hat{J}$ is chosen as a primary dependent variable in developing the governing system of equations. To this end, (25) and (26) become

$$
\begin{gathered}
\sigma_{r r}^{\mathrm{sat}}=\Phi+K\left[1+\alpha \lambda^{2}\left(1+\hat{\lambda}^{2}\right)\right] \frac{\hat{J}}{\lambda \hat{\lambda}^{2}}, \\
\sigma_{\theta \theta}^{\mathrm{sat}}=\Phi+K\left[\left(1+\lambda^{2} \alpha\right)\left(\hat{\lambda}^{2}+\left(f g^{\prime}\right)^{2}\right)+\hat{J}^{2}\right] \frac{1}{\lambda \hat{J}} .
\end{gathered}
$$

When $f g^{\prime}$ is substituted from (43), the right-hand sides of (44) and (45) become functions of $\hat{J}$ and $\hat{\lambda}^{2}$ as well as of $\hat{r}$. The problem is now formulated as a system of two first order nonlinear ordinary differential equations for $\hat{J}$ and $\hat{\lambda}^{2}$.

The first equation is a compatibility condition obtained by differentiating (21) with respect to $\hat{r}$. Using (22), this can be written in the form

$$
\frac{\mathrm{d}(\hat{\lambda})^{2}}{\mathrm{~d} \hat{r}}=\frac{2}{\hat{r}}\left(\hat{J}-\hat{\lambda}^{2}\right)
$$


Next let (44) and (45) be substituted into (32). The resulting equation has the form,

$$
\frac{\mathrm{d} \hat{J}}{\mathrm{~d} \hat{r}}=-\frac{\left[Z_{3}\left(\hat{\lambda}^{2}, \hat{J}, \hat{r}\right)+2\left(\hat{J}-\hat{\lambda}^{2}\right) Z_{2}\left(\hat{\lambda}^{2}, \hat{J}\right)\right]}{\hat{r} Z_{1}\left(\hat{\Lambda}^{2}, \hat{J}\right)}
$$

where use has been made of (46), and the functions $Z_{1}, Z_{2}$ and $Z_{3}$ are defined by the relations

$$
\begin{gathered}
Z_{1}\left(\hat{\lambda}^{2}, \hat{J}\right)=-\frac{M_{c}}{\rho_{0}^{\mathrm{s}} V_{1}}\left(2 \chi-\frac{1}{1-v}\right) \frac{\hat{\lambda}^{4}}{\lambda^{5} \hat{J}^{3}}+\left[1+\alpha \lambda^{2}\left(1+\hat{\lambda}^{2}\right)\right] \hat{\lambda}^{2}, \\
Z_{2}\left(\hat{\lambda}^{2}, \hat{J}\right)=-\left[1+\alpha \hat{\lambda}^{2}\left(1+\hat{\lambda}^{2}\right)\right] \hat{\jmath}+\alpha \lambda^{2} \hat{J} \hat{\lambda}^{2} \\
Z_{3}\left(\hat{\lambda}^{2}, \hat{J}, \hat{r}\right)=\left(1+\lambda^{2} \alpha\right) \hat{\lambda}^{2}\left[\frac{\hat{J}^{2}}{\hat{\lambda}^{2}}-\hat{\lambda}^{2}-\left(f g^{\prime}\right)^{2}\right]
\end{gathered}
$$

where $v$ is defined in (38) and $f g^{\prime}$ is given by (43).

Let $\bar{r}$ and $\bar{f}$ be dimensionless radii defined by

$$
\bar{r}=\hat{r} / \lambda R_{\mathrm{i}}, \quad \bar{f}=f / \lambda R_{\mathrm{i}} .
$$

Then $\vec{r} \ni\left[1, R_{\mathrm{o}} / R_{\mathrm{i}}\right]$. When these are introduced into (46)-(50), $f^{\prime}, \hat{\lambda}, \hat{J}$ and the equations have the same form except that $\hat{r}$ is replaced by $\bar{r}, f$ is replaced by $\bar{f}$ and (43) becomes

$$
f g^{\prime}=\frac{M^{*} \lambda}{1+\lambda^{2} \alpha} \frac{1}{\bar{r}^{2}} \frac{1}{\hat{\lambda}}
$$

where $M^{*}$ is a dimensionless moment per unit length and is defined by the relation

$$
M^{*}=\frac{M}{2 \pi \lambda^{2} R_{\mathrm{i}}^{2} K} .
$$

The boundary conditions for (46)-(50) are obtained from (34) and are

$$
\hat{\lambda}^{2}=1, \bar{r}=1, \text { and } \bar{r}=R_{\mathrm{o}} / R_{\mathrm{i}} .
$$

Once $\hat{\lambda}^{2}$ and $\hat{J}$ have been determined, $g(\bar{r})$ can be obtained by integrating (52), subject to boundary condition (35). In terms of the dimensionless quantities, the relation for $g(\bar{r})$ is given by

$$
g(\bar{r})=\frac{M^{*} \lambda}{1+\lambda^{2} \alpha} \int_{1}^{\bar{r}} \frac{\mathrm{d} x}{x^{3} \hat{\lambda}^{2}(x)}
$$

\section{NUMERICAL SOLUTION}

The boundary value problem was solved by a numerical method. The differential equations were integrated using a fourth order Runga-Kutta method. A one-dimensional shooting method was used to select $\hat{J}$ at $\bar{r}=1$ so as to satisfy the boundary condition at $\bar{r}=R_{\mathrm{o}} / R_{\mathrm{i}}$. The material parameters were the same as in previous work (cf. [1-3]) and correspond to a vulcanized rubber-toluene mixture: $R=8.317 \times 10^{7}$ dyne-cm $/ \mathrm{mol} \mathrm{K}, T=303.16 \mathrm{~K}, \quad V_{1}=$ $106 \mathrm{~cm}^{3} / \mathrm{mol}, \rho_{0}^{\mathrm{s}}=0.862 \mathrm{~g} / \mathrm{cm}^{3}, \chi=0.425, M_{\mathrm{c}}=9151 \mathrm{~g} / \mathrm{mol}$. Calculations were carried out for $\alpha=0$ and $\alpha=1$. For the unswollen rubber cylinder $R_{\mathrm{o}} / R_{\mathrm{i}}=2$.

When $\alpha=0$, it was found from (40) that $\lambda=1.723$ which corresponds to a ratio of the swollen unsheared state volume to its initial volume of $\lambda^{3}=5.115$. When $\alpha=1, \lambda=1.388$ and 


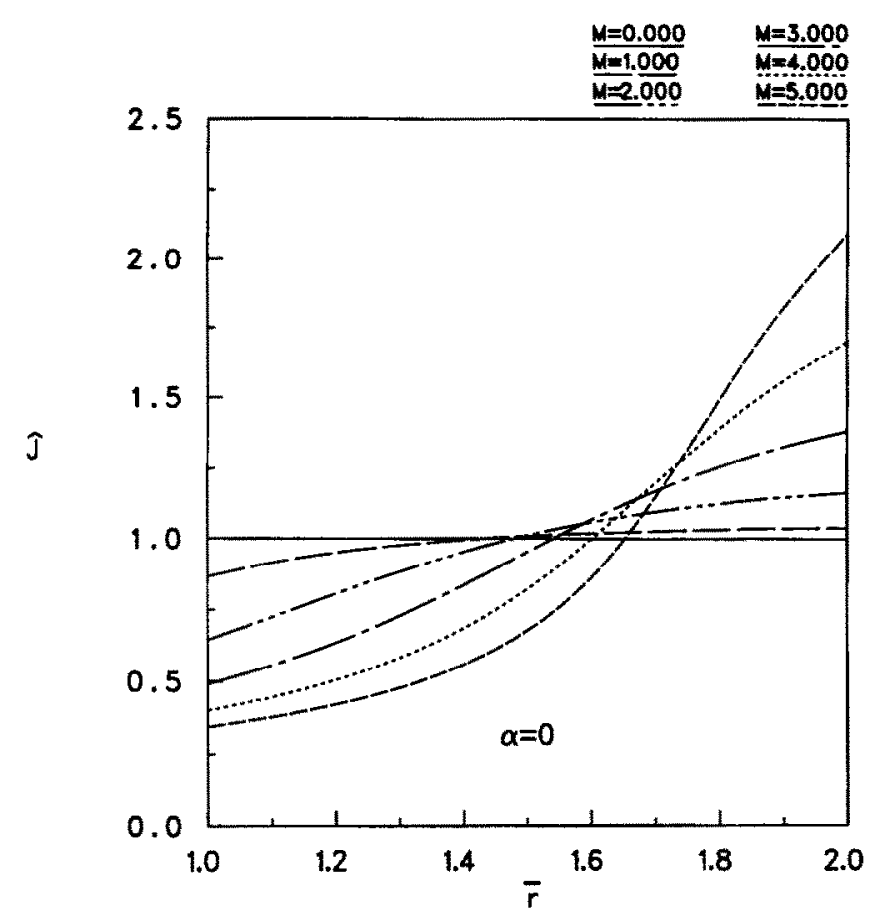

Fig. 1. $\hat{J}$ vs $\ddot{r}$ for $\alpha=0$.

the volume ratio is 2.674 . Thus, when $A_{0}^{\mathrm{e}}$ has the Mooney form, the predicted amount of swelling is reduced.

It is clear from the numerical results that the shear deformation does indeed cause local volume changes and redistribution of the liquid. Figures 1-4 present results for the case when $\alpha=0$. Figure 1 shows the radial variation of $\hat{J}$. The volume decreases from that in the swollen unsheared state near the inner portion of the cylinder and increases near the outer portion. The

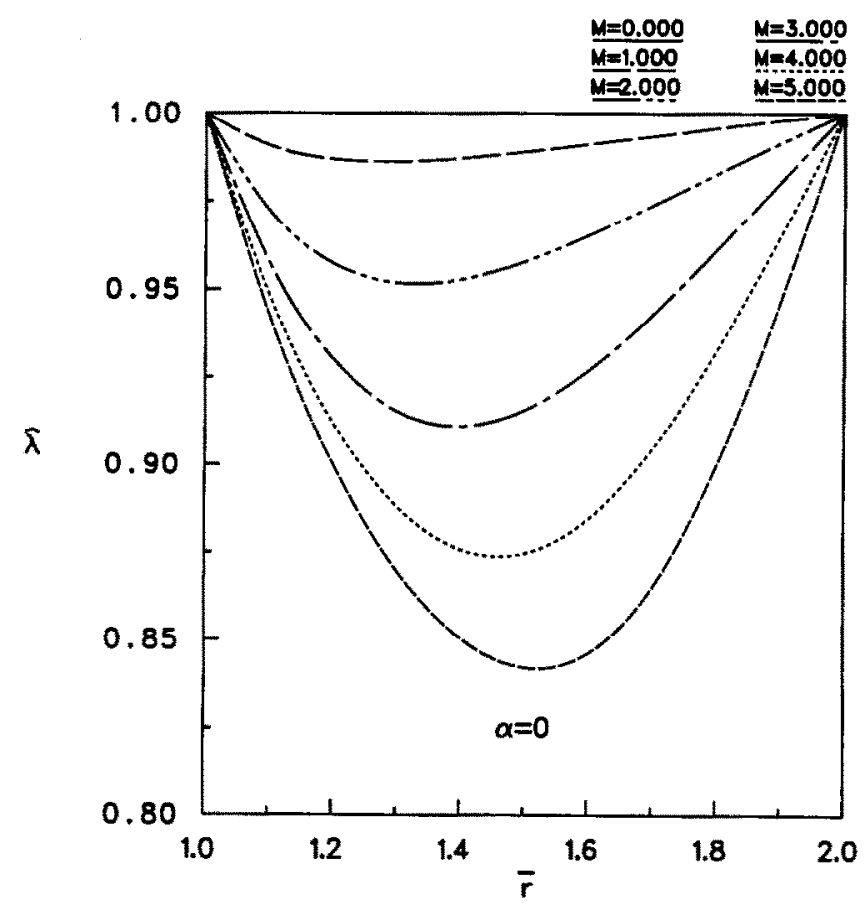

Fig. 2. $\hat{\lambda}$ vs $\bar{r}$ for $\alpha=0$. 


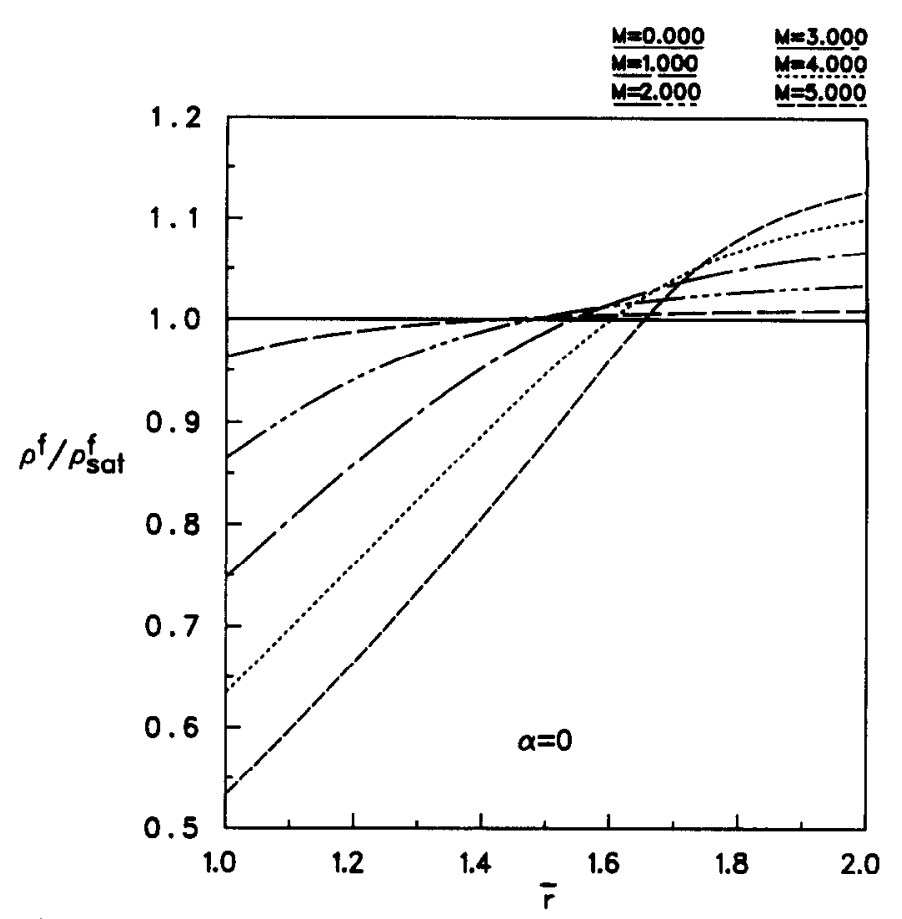

Fig. 3. $\rho^{\mathrm{f}} / \rho_{\mathrm{sat}}^{\mathrm{f}}$ vs $\bar{r}$ for $\alpha=0$. $\rho_{\mathrm{sat}}^{\mathrm{f}}$ is the fluid density in the swollen unsheared state.

change increases with the applied moment. As seen from Fig. 2, this volume change is produced when all cylindrical surfaces move to smaller radii. The volume contained between two cylindrical surfaces thereby increases in the outer portion and decreases near the inner portion. This is consistent with the plot shown in Fig. 3 of the radial variation of the current fluid density normalized by its value in the swollen unsheared state. As the moment increases, fluid leaves the mixture in the inner region, causing a local volume decrease, and moves to the

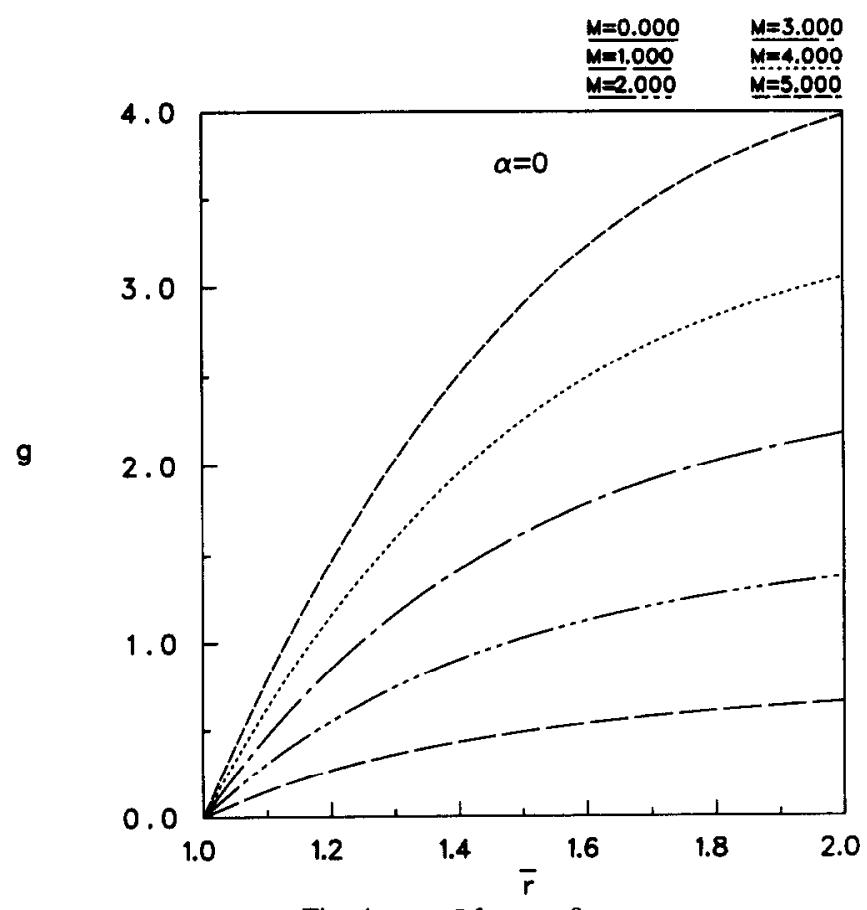

Fig. 4. $g$ vs $\bar{r}$ for $\alpha=0$. 


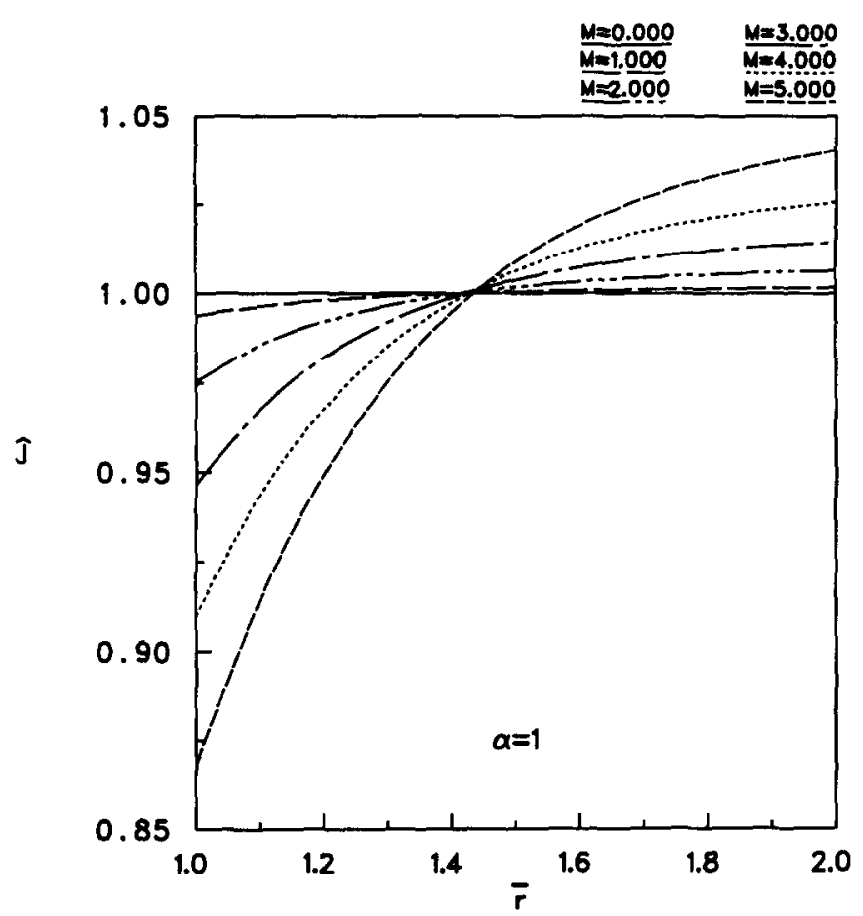

Fig. 5. $\hat{J}$ vs $\bar{r}$ for $\alpha=1$.

outer region, causing a local volume increase. The rotation $g(\bar{r})$ of the cylindrical surfaces is shown in Fig. 4. The rotation increases rapidly near the inner surface and then more gradually in the outer region. This is a consequence of the inner region being one of the higher shear stress, as indicated in (42), and the relation between the local shear deformation and the slope of the rotation as seen from (20).

Distributions of local volume ratio, stretch ratio, and fluid density ratio, when $\alpha=1$, are

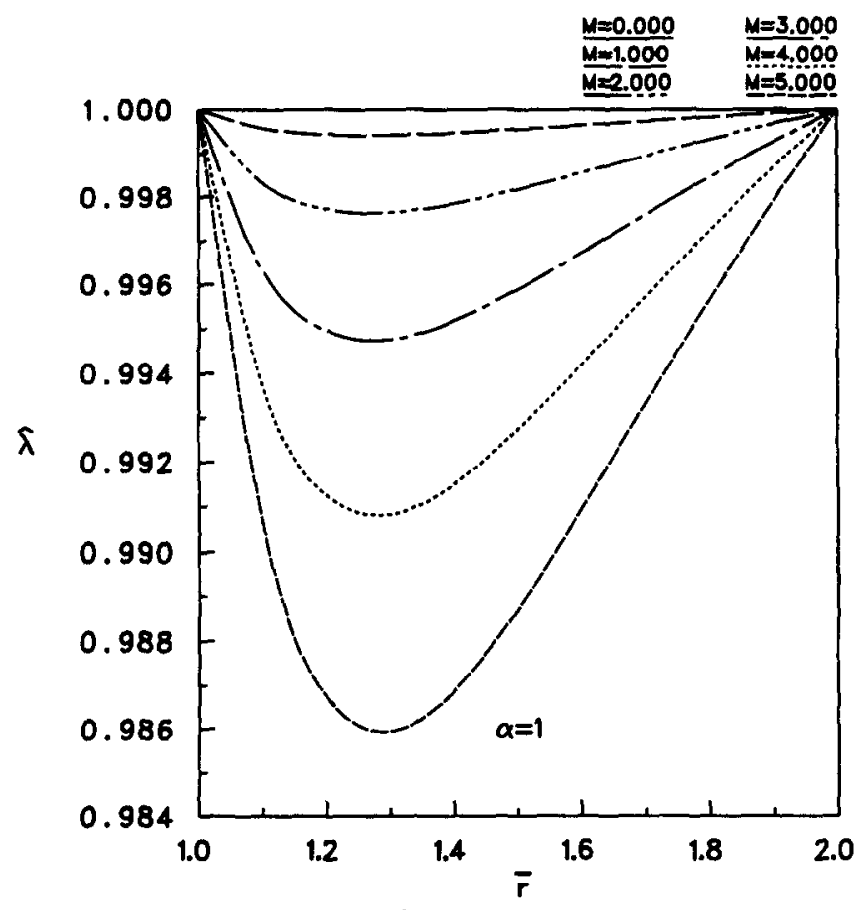

Fig. 6. $\hat{\lambda}$ vs $\bar{r}$ for $\alpha=1$. 


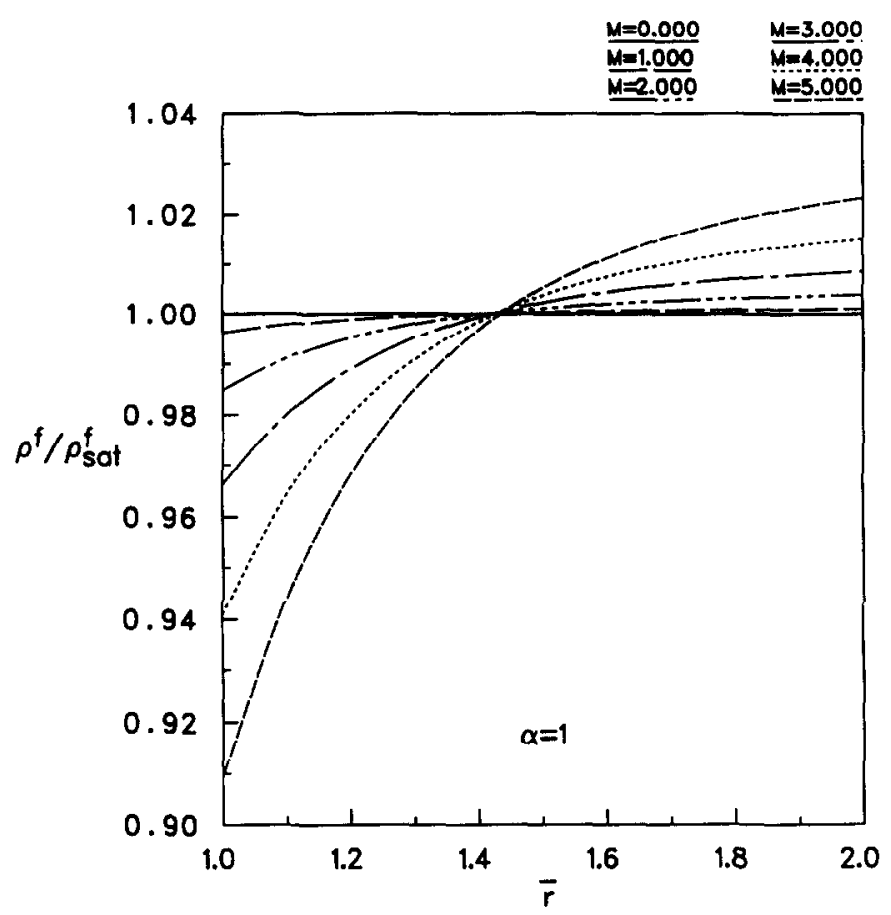

Fig. 7. $\rho^{\mathrm{f}} / \rho_{\mathrm{sat}}^{\mathrm{f}}$ vs $\bar{r}$ for $\alpha=1$. $\rho_{\mathrm{sat}}^{\mathrm{f}}$ is the fluid density in the swollen unsheared state.

shown in Figs 5-7, respectively. The distributions have the same general features as in the case when $\alpha=0$. However, when $A_{0}^{\mathrm{c}}$ has the Mooney form, the changes in the mixture caused by shearing are much smaller than in the neo-Hookean case. The relation between the moment and the rotation of the outer surface is shown in Fig. 8 , for $\alpha=0$ and 1 . The relation is slightly softer than a linear one and gives no indication of the substantial changes induced in the mixture by the shear deformation.

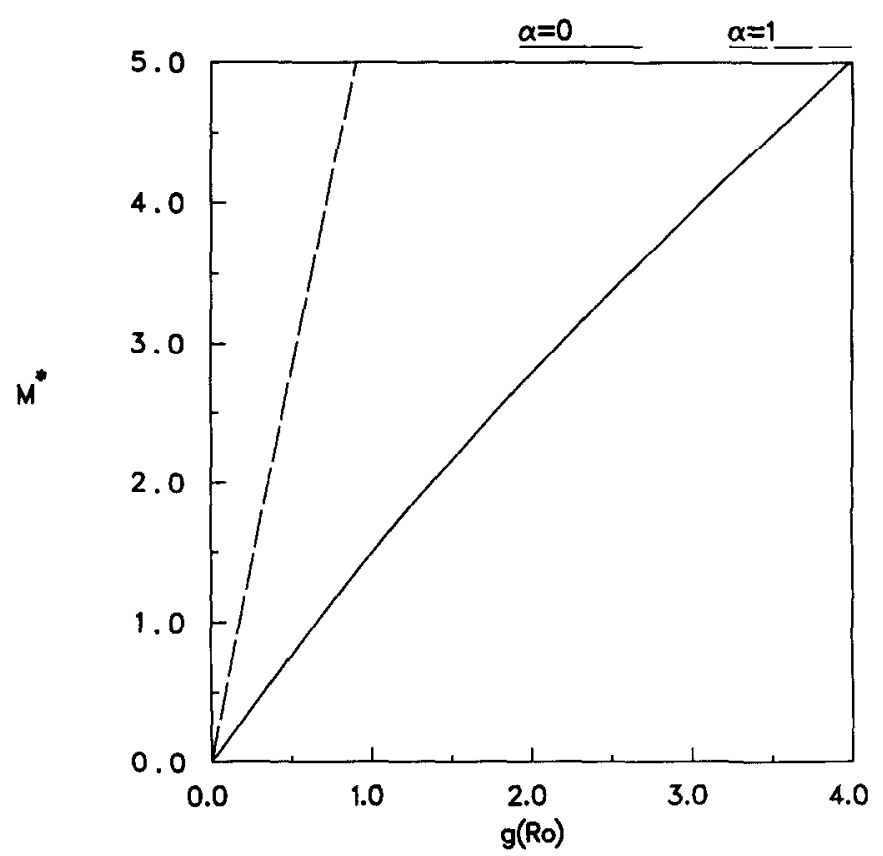

Fig. 8. Dimensionless moment $M^{*}$ vs angle of rotation of outer cylinder for $\alpha=0$ and 1 . 
Acknowledgement-The authors wish to thank William K. Waldron for computational assistance.

\section{REFERENCES}

[1] J. J. SHI, K. R. RAJAGOPAL and A. S. WINEMAN, Int. J. Engng Sci. 9, 871 (1981).

[2] M. V. GANDHI, A. S. WINEMAN and K. R. RAJAGOPAL, Int. J. Engng Sci. 25, 1441 (1987).

[3] F. DAI, K. R. RAJAGOPAL and A. S. WINEMAN, Int. J. Engng Sci. 29, 419 (1991).

[4] K. R. RAJAGOPAL and A. S. WINEMAN, Recent Developments in Structured Continua (edited by D. DEKEE and P. N. KALONI), Vol. II. Pitman Research Notes in Mathematics series, Longman Scientific and Technical, Harlow, U.K.

[5] M. V. GANDHI, M. USMAN, A. S. WINEMAN and K. R. RAJAGOPAL, Acta Mechanica 79, 81 (1989).

[6] R. M. BOWEN, Continuum Physics (Edited by A. C. ERINGEN), Vol. III. Academic Press, New York (1975).

[7] R. J. ATKIN and R. E. CRAINE, Q. J. Mech. Appl. Math. XXIX, 209 (1976).

[8] L. R. G. TRELOAR, The Physics of Rubber Elasticity. Oxford Univ. Press, Oxford (1975).

[9] N. MILLS, Int. J. Engng Sci. 4, 97 (1966)

[10] A. E. GREEN and P. M. NAGHDI, Q. J. Mech, Appl. Math. 22, 427 (1969).

[11] K. R. RAJAGOPAL, A. S. WINEMAN and M. GANDHI Int. J. Engng Sci. 24, 1453 (1986).

[12] M. V. GANDHI and M. USMAN, Int. J. Engng Sci. 27, 539 (1989). 